Artigo Original

\title{
Satisfação corporal em acadêmicos de Educação Física: proposta de um questionário
}

\author{
Fernando Luiz Cardoso \\ Cinara Sacomori \\ Fabiana Flores Sperandio \\ Ana Paula Krüger
}

Centro de Ciências da Saúde e do Esporte da UDESC, Florianópolis, SC, Brasil

\begin{abstract}
Resumo: A satisfação corporal pode ser dimensionada em: imagem corporal, satisfação com o peso e catexia corporal. Avaliou-se a satisfação corporal de 302 acadêmicos de Educação Física e sugeriu-se um questionário. Os estudantes se consideraram de médio a muito satisfeitos com seus corpos. Os homens apresentaram maiores médias para: "quanto acha seu corpo proporcional" e "quanto está satisfeito com seu corpo". Já as mulheres para: "quanto olha seu corpo no espelho", "quanto toca o corpo de forma geral", "quanto percebe que os outros acham sexy" e "quanto gostaria de ser mais magra". A análise fatorial confirmou o modelo e obteve-se $\alpha=, 757$.
\end{abstract}

Palavras-chave: Satisfação Pessoal. Corpo. Educação Física.

\section{Body satisfaction in Physical Education undergraduate students: questionnaire proposal}

Abstract: Body satisfaction can be divided into: body image, satisfaction with weight and body cathexis. Body satisfaction of 302 Physical Education undergraduate students was evaluated and a questionnaire was proposed. Students self-evaluated as medium to very satisfied with their bodies. Men had higher means $(p<.05)$ regards "to feel their body proportionate" and "being satisfied with their bodies". And women "look more their bodies at the mirror", "touch more the bodies in general", "find themselves more sexy" and "would like to be thinner". The factor analysis confirmed the model and got a $\alpha=.757$.

Keywords: Personal Satisfaction. Body. Physical Education.

\section{Introdução}

Os profissionais de Educação Física trabalham diretamente com a promoção da saúde, mediante as múltiplas expressões e manifestações, tanto do movimento humano, quanto do corpo em relação ao meio. Entende-se que ainda existem aspectos inexplorados e, até mesmo, controversos em relação à variedade e diversidade de intervenções destes profissionais. É preciso considerar, além disso, a existência de uma interligação e de uma justaposição dos múltiplos papéis desempenhados por estes profissionais na busca da promoção da saúde.

À priori são estes profissionais, no cotidiano de suas atividades, que percebem indagações de seus clientes em relação à beleza, à aparência e à imagem corporal. Tais indagações, geralmente são repletas de inseguranças e expectativas de desempenho físico e estético, fruto de imposições construídas sócio-culturalmente (TAVARES, 2003; PETROSKI et al, 2009; TIGGEMANN, 2004). Entretanto, conforme observado por Philips e Drummond (2001), esses profissionais podem ter as mesmas expectativas e inseguranças dos seus alunos e clientes, ou mais que isso, podem projetar metas de trabalho dissonantes para os mesmos, o que poderá dificultar uma boa relação entre professor e aluno/cliente.

Cotejando tais informações, Frost e McKelvie (2005) consideraram que a satisfação corporal está alicerçada por três componentes, quais sejam, a imagem corporal, satisfação com o peso e a catexia corporal. Os autores consideram que a imagem corporal é o quão perto ou distante a imagem corporal atual está daquela considerada ideal pelo indivíduo. A satisfação com o peso corporal é descrita como o quão próximo o peso corporal de alguém está do peso considerado ideal por este. Por fim, catexia corporal diz respeito à satisfação que alguém tem em relação a uma parte específica ou aspecto do próprio corpo.

Por conseguinte, estes três elementos (satisfação, imagem corporal e catexia corporal) podem influenciar o desenvolvimento físicoemocional a partir da forma como alguém se vê e como avalia o seu próprio desempenho. Muitas 
destas questões são estudadas sob a ótica da identidade de gênero, do sexo, da idade e maturação biológica (KOSTANKI; GULLONE, 1998; ATA et al 2007; KASHUBECK-WEST et al., 2005; TIGGEMANN, 2004; DOHNT; TIGGEMANN, 2006; BEARMAN et al., 2006).

O processo de representação do corpo na mente humana constitui a imagem corporal (TAVARES, 2003). A este respeito, Huang et al. (2007) consideram imagem corporal como um conjunto das auto-percepções corporais e comportamentais que o indivíduo tem de si, relacionando-se à auto-estima, segurança, alimentação e atividade física, experiências sexuais e estabilidade emocional.

Diante disto, a construção da imagem corporal ocorre por influência de contextos culturais, sociais e psicológicos (RICCIARDELLI; MCCABE, 2003; LEE et al 2009). É, portanto, considerada como um fenômeno complexo e multidimensional (LEE et al 2009), cujos fatores relacionados são o peso corporal (JONES, 2004; STICE; WHITTENTON, 2002), a idade (PUHL; BROWNELL, 2001; VANDERWAL; THELEN, 2000; DOHNT; TIGGEMANN, 2006; BEARMAN et al., 2006) e o gênero (HARGREAVES; TIGGEMANN, 2004; JONES, 2004; RICCIARDELLI; MCCABE, 2003; BEARMAN et al., 2006).

No que se refere às diferenças da imagem corporal entre homens e mulheres, estas tendem a supervalorizar o baixo peso, contrariamente aos homens que apreciam o volume muscular desde o período de transição da adolescência até a idade adulta (NEUMAN et al 2006). Segundo Tiggeman (2004), a preocupação com a imagem corporal feminina parece decrescer com a idade, embora episódios importantes como a puberdade, a gestação e a menopausa possam operar negativamente para a satisfação corporal.

Uma imagem corporal negativa ou a não satisfação com o corpo mantém associação com o tamanho/forma e o peso corporal (obesidade ou sobrepeso) (JONES, 2004; LEE et al 2009), sendo sugerido pelos autores como indicadores de alterações no comportamento estético, na freqüência de exercícios (LEE et al 2009) e no padrão alimentar (NEUMAN et al 2006).

Dada a complexidade e importância de se mensurar a satisfação corporal, Lee et al (2009), demonstram que os instrumentos até então publicados, independentemente dos itens presentes no modelo elegido, destinam-se a avaliar uma parte específica do corpo ou a combinação delas entre si.

Estudos prévios com alunos de graduação identificaram atitudes negativas em relação ao corpo e ao peso (JUNG et al 2001; LOWERY et al., 2005; TIGGEMANN; WILLIAMSON, 2000). Outro estudo ainda investigou as percepções de imagem corporal, satisfação corporal e prática de exercícios em instrutores homens de academia e explorou o impacto destes aspectos na prática profissional dos mesmos. Esses profissionais demonstraram confiar em suas capacidades de perder ou manter o peso corporal a partir do exercício físico, o que foi relacionado com um senso de controle corporal (PHILIPSPHILIPS; DRUMMOND, 2001). Além disso, outro estudo revelou que homens que se exercitavam muito reportavam maior satisfação com aspectos dos seus corpos que aqueles que se exercitavam pouco (FROST; MCKELVIE, 2005).

Nesse contexto, os objetivos deste estudo foram avaliar a satisfação corporal de acadêmicos de Educação Física, homens e mulheres, e propor um breve questionário para avaliar a satisfação corporal. A confiabilidade interna e a validade de construto foram, igualmente, testadas no estudo.

\section{Método}

Esta é uma pesquisa não-probabilística descritiva correlacional. A coleta de dados faz parte de uma pesquisa mais ampla, aprovada pelo Comitê de Ética em Pesquisa da UDESC, sob o número de referência 40/05. O comitê de ética dispensou a utilização do Termo de Consentimento Livre e Esclarecido (TCLE). Tal atitude evitou a identificação dos participantes que preencheram o QIC de forma anônima. Antes do preenchimento em grandes grupos, este termo de consentimento foi lido publicamente e perguntouse quem gostaria de tomar parte nessa pesquisa que abordava questões da intimidade e privacidade. Após o preenchimento inominado e individual os participantes dobravam 0 questionário e o depositavam em uma urna lacrada que só foi aberta ao final de cada coleta

Os dados foram coletados no curso de graduação em Educação Física do Centro de Ciências da Saúde e Esportes - CEFID da Universidade do Estado de Santa Catarina - 
UDESC, em Florianópolis, no período de novembro de 2007 a março de 2008.

\section{Participantes}

Participaram do estudo 157 homens e 145 mulheres, matriculados regularmente no curso de graduação em Educação Física, sendo que 132 homens (84,10\%) e 109 mulheres (75,2\%) praticavam atividade física regularmente. Apenas sete homens $(4,5 \%)$ e 14 mulheres $(9,7 \%)$ eram casados ou viviam com um companheiro. Os detalhes sobre o perfil dos participantes podem ser vistos na Tabela 1.

Tabela 1- Perfil sócio-antropométrico dos homens e mulheres participantes.

\begin{tabular}{lcccccc}
\hline \multirow{2}{*}{ Parâmetros } & \multicolumn{2}{c}{ Homens $(\mathrm{N}=157)$} & \multicolumn{2}{c}{ Mulheres $(\mathrm{N}=145)$} & \multirow{2}{*}{ t test } & \multirow{2}{*}{$p$} \\
& $X$ & $s d$ & $X$ & $S d$ & & \\
\hline Idade $^{1}$ & 21,44 & 2,76 & 21,20 & 2,94 & 0,73 & n.s. \\
Altura $^{2}$ & 1,77 & 0,06 & 1,65 & 0,05 & 17,19 & .001 \\
Peso $^{3}$ & 75,44 & 10,31 & 57,67 & 7,36 & 17,06 & .001 \\
Nível de atividade física $^{4}$ & 4,36 & 1,35 & 3,41 & 1,46 & 5,43 & .001 \\
Itens de consumo $^{5}$ & 2,68 & 1,00 & 2,80 & 1,08 & $-0,98$ & n.s. \\
\hline
\end{tabular}

1. Questão: Idade (em anos): __ anos. Media aritmética da idade dos sujeitos.

2. Questão: Altura (em centímetros): $\quad \mathrm{cm}$. Media aritmética da altura dos sujeitos.

3. Questão: Peso (em quilos): __ kg. Media aritmética do peso dos sujeitos.

4. Questão: O quanto ativo você se considera em termos de atividade física ou exercício físico? Media aritmética de uma Escala Likert de (0-nada, 1-pouco, 2-razoavelmente, 3-medianamente, 4satisfatoriamente, 5-bem, 6-muito).

5. Questão: Quais desses itens você tem em casa? ( ) Ar Condicionado, ( ) TV a cabo, ( ) Carro, ( ) Computador. Media aritmética dos itens de consumo que possua em casa.

\section{Instrumento}

Utilizaram-se 14 itens do Questionário de Identidade Corporal (QIC), desenvolvido pelo Laboratório de Gênero, Sexualidade e Corporeidade do CEFID - UDESC. Em sua totalidade, este é composto por perguntas relativas à intensidade (pouco, médio, muito) de alguns eventos mensurados por meio de uma escala análogo visual do tipo Likert. $O$ instrumento consta de uma parte inicial com perguntas gerais sobre como os indivíduos se classificam em termos de sexo, gênero, orientação sexual, estado civil, atividade física, escolaridade e demais aspectos sociais e uma segunda parte constituída de 7 escalas, a saber: 1. Relação com o corpo; $2 . \quad$ Percepção corporal; 3. Comportamento sexual; 4. Pré-disposição sexual; 5. Orientação sexual; 6. Percepção do prazer sexual; e 7. Satisfação sexual. Esse instrumento tem como objetivo maior, entender a relação dos sujeitos com a sua sexualidade e corporeidade. $O$ QIC é um instrumento de caráter subjetivo, portanto, baseado na mensuração do auto-relato e previamente utilizado em outros estudos (CARDOSO et al., 2009; CARDOSO et al., 2010; $\underline{\text { SACOMORI }}$ et al. 2010).

Utilizaram-se apenas as perguntas do QIC relativas aos aspectos da satisfação corporal: catexia corporal, imagem corporal e satisfação com o peso, provenientes do modelo de Frost e
Mckelvie (2005). Após, realizou-se uma análise fatorial exploratória dos itens pré-selecionados e identificados no QIC e excluíram-se aqueles que não carregaram adequadamente nos três fatores provenientes deste modelo.

\section{Coleta dos dados}

Participaram da coleta dos dados três mestrandos e quatro bolsistas de iniciação à pesquisa que abordavam os indivíduos explicando os objetivos da pesquisa e solicitavam a participação anônima. Após o preenchimento, os questionários foram depositados em uma urna lacrada, a qual só foi aberta após o término da coleta, conforme a orientação do Comitê de Ética.

\section{Organização e análise dos dados}

Os dados foram organizados no programa estatístico SPSS versão 13.0, onde se usou o Teste-t para amostras independentes (para diagnosticar possíveis diferenças entre homens e mulheres). Para investigar a estrutura fatorial dos itens que corresponderiam à tripartição proposta por Frost e McKelvie (2005) utilizou-se análise fatorial exploratória com rotação oblíqua (Direct Oblimin). A consistência interna de cada dimensão foi analisada por meil do coeficiente alfa de Cronbach. O Teste de correlação de Pearson foi realizado para identificar possíveis correlações dentre outras variáveis do QIC com o construto satisfação corporal. 


\section{Resultados}

Satisfação Corporal de Acadêmicos de Educação Física

Observa-se (tabela 2) que, no geral, os acadêmicos de Educação Física estavam de médio a muito satisfeitos com seus corpos, uma vez que a média para a maioria das variáveis foi maior que 3 (quando valores próximos a 6 eram indicativos de uma melhor satisfação corporal).

Tabela 2- Diferenças observadas entre homens e mulheres a partir do Questionário

\begin{tabular}{|c|c|c|c|c|c|c|}
\hline \multirow[b]{2}{*}{ Itens do Questionário de Satisfação Corporal } & \multicolumn{2}{|c|}{ Homens } & \multicolumn{2}{|c|}{ Mulheres } & \multirow[t]{2}{*}{$\mathbf{t}$} & \multirow[t]{2}{*}{$\mathbf{p}$} \\
\hline & Média & Sd & Média & Sd & & \\
\hline O quanto olha seu corpo inteiro no espelho & 3,83 & 42 & 4,85 & 1,30 & $-6,51$ &, 000 \\
\hline & $2, \varepsilon$ & & 3,2 & & $-2,78$ &, 006 \\
\hline O quanto toca seu corpo de form & 3,8 & 1,44 & 4,21 & 1,28 & $-2,16$ & ,032 \\
\hline gosta do seu cabelo & & & 4,34 & & $-0,25$ & ,806 \\
\hline $\mathrm{Oq}$ & $4, \varepsilon$ & & 4,86 & & $-0,05$ & ,959 \\
\hline a cor da sua & 5,12 & 1,12 & 4,59 & 1,50 & 3,46 & 001 \\
\hline $\mathrm{Og}$ & & 1,32 & 4,60 & & $-0,17$ & 864 \\
\hline $\mathrm{Oq}$ & 4 , & 1, & 4,10 & 1 & 0,22 & ,820 \\
\hline $\mathrm{Ogu}$ & 3,8 & 1,10 & 3,73 & 1,10 & 1,23 & ,221 \\
\hline O quanto acha seu corpo proporcio & 4,13 & 1,10 & 3,87 & 1,22 & 1,97 & 049 \\
\hline $\mathrm{Oq}$ & 3,7 & 1,21 & 3,69 & & 0,41 & ,683 \\
\hline $\mathrm{OO}$ & 4, & 1,32 & 3,56 & 1,44 & 2,73 &, 007 \\
\hline O quanto percebe que os outro & 3,21 & 1,23 & 3,60 & 1,31 & $-2,68$ &, 008 \\
\hline O quanto gostaria de ser mais magro & 1,87 & 1,8 & 3,21 & 2.07 & $-5,96$ &, 000 \\
\hline
\end{tabular}

Percebeu-se algumas diferenças entre homens e mulheres com relação a alguns aspectos da satisfação corporal. Os homens apresentaram maiores médias $(p<, 05)$ nas variáveis: "o quanto gosta da cor da sua pele", "o quanto acha seu corpo proporcional" e "o quanto está satisfeito com seu corpo". Já as mulheres apresentaram maiores médias $(p<, 05)$ nas variáveis: "o quanto olha seu corpo inteiro no espelho", "o quanto se auto-massageia", "o quanto toca o corpo de forma geral", "o quanto percebe que os outros o acham sexy" e "o quanto gostaria de ser mais magra".

Foram obtidos índices de imagem corporal, catexia corporal e satisfação com o peso a partir do somatório das escalas pertencentes a cada um deles. Posteriormente, esses índices foram submetidos ao teste de correlação com outras variáveis do QIC e controle.

Figura 1- Correlações no grupo de homens

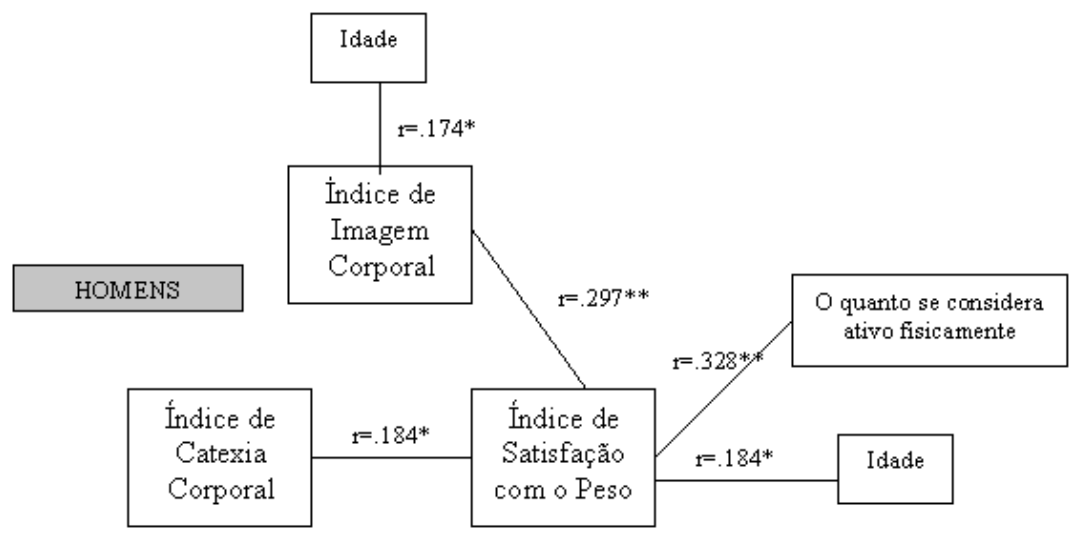

Assim, obteve-se, para os homens (figura 1), correlação positiva entre a idade e os índices de imagem corporal e satisfação com o peso, este último também esteve positivamente correlacionado com "o quanto se considerava fisicamente ativo". O índice de satisfação com o peso correlacionou-se com os outros dois índices, porém não houve correlação entre os índices de imagem e catexia corporal. 
Para as mulheres (figura 2), a idade correlacionou-se positivamente com os três índices e o índice de satisfação com o peso apresentou correlação negativa com o índice de massa corporal (IMC). Diferente do que ocorreu com os homens, houve correlação positiva e mais forte entre os três índices de satisfação corporal.

Figura 2- Correlações no grupo de mulheres

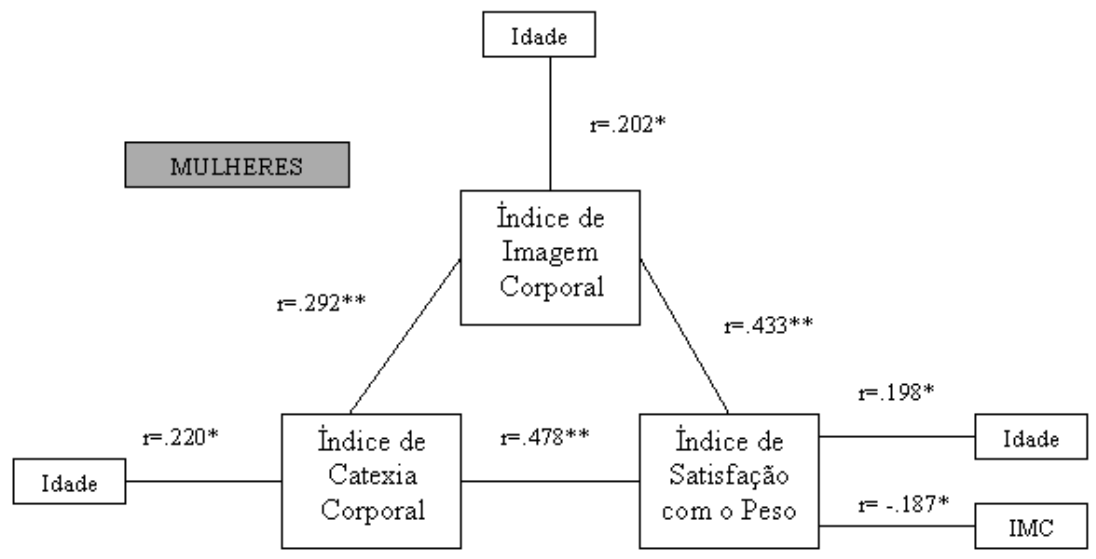

\section{Consistência Interna do questionário de Satisfação Corporal proposto}

Quatorze itens do QIC (do total de 135) que tinham relação com 0 construto satisfação corporal foram pré-selecionados pelos autores. Realizou-se uma análise fatorial pelo método de fatoração pelos eixos principais (rotação direct oblimin). Os itens carregaram significativamente em três fatores principais, sendo que o padrão de distribuição da análise fatorial foi similar para homens e mulheres (tabela 3). A única exceção foi para o fator satisfação com o peso, em que o item "o quanto gostaria de ser mais magro" não carregou para os homens.

Tabela 3- Análise fatorial do construto Satisfação Corporal

\begin{tabular}{|c|c|c|c|c|c|c|}
\hline \multirow[t]{2}{*}{ Itens do QIC } & \multicolumn{3}{|c|}{$\begin{array}{l}\text { Fatores da extração } \\
\text { (homens) }\end{array}$} & \multicolumn{3}{|c|}{$\begin{array}{l}\text { Fatores da extração } \\
\text { (mulheres) }\end{array}$} \\
\hline & $\begin{array}{c}1 \\
\text { (Imagem } \\
\text { Corporal) }\end{array}$ & $\begin{array}{c}2 \\
\text { (Catexia } \\
\text { Corporal) }\end{array}$ & $\begin{array}{c}3 \\
\text { (Satisfação } \\
\text { com peso) }\end{array}$ & $\begin{array}{c}1 \\
\text { (Imagem } \\
\text { Corporal) }\end{array}$ & $\begin{array}{c}2 \\
\text { (Catexia } \\
\text { Corporal) }\end{array}$ & $\begin{array}{c}3 \\
\text { (Satisfação } \\
\text { com peso) }\end{array}$ \\
\hline $\begin{array}{l}\text { 1. O quanto olha o corpo inteiro no } \\
\text { espelho }\end{array}$ & , 8 & & &,- 6 & & \\
\hline 2. O quanto se auto-massageia & 73 & & &,- 8 & & \\
\hline $\begin{array}{l}\text { 3. O quanto toca o corpo de forma } \\
\text { geral }\end{array}$ & 8 & & &,- 7 & & \\
\hline 4. O quanto gosta do seu cabelo & & ,80 & & & ,73 & \\
\hline 5. O quanto gosta da cor dos olhos & & ,75 & & &, 74 & \\
\hline 6. O quanto gosta da cor da pele & &, 52 & & & 58 & \\
\hline 7. O quanto gosta do seu rosto & & 64 & & & ,74 & \\
\hline $\begin{array}{l}\text { 8. O quanto percebe que causa uma } \\
\text { boa impressão }\end{array}$ & & & ,707 & & & ,656 \\
\hline 9. O quanto acha o corpo bonito & & & ,856 & & & ,769 \\
\hline $\begin{array}{l}\text { 10. O quanto acha o corpo } \\
\text { proporcional }\end{array}$ & & & ,783 & & & ,742 \\
\hline 11. O quanto acha o corpo sensual & & & ,782 & & & ,699 \\
\hline $\begin{array}{l}\text { 12. O quanto está satisfeito com o } \\
\text { seu corpo }\end{array}$ & & &, 684 & & & ,841 \\
\hline $\begin{array}{l}\text { 13. O quanto percebe que os outros } \\
\text { o acham sexy }\end{array}$ & & & ,731 & & &, 543 \\
\hline $\begin{array}{l}\text { 14. O quanto gostaria de ser mais } \\
\text { magro(a) }\end{array}$ & & & $\star * * *$ & & &,- 771 \\
\hline $\begin{array}{l}\text { Percentual da variância } \\
\text { Alfa de Crombach }\end{array}$ & $\begin{aligned} 13, \\
, 7\end{aligned}$ & $\begin{aligned} 13,6 \\
, 67\end{aligned}$ & $\begin{array}{c}31,1 \% \\
, 869\end{array}$ & $\begin{array}{l}9,9 \\
, 68\end{array}$ & $\begin{array}{r}13,2 \\
, 70\end{array}$ & $\begin{array}{c}40,5 \% \\
, 898\end{array}$ \\
\hline
\end{tabular}

O teste de Keiser-Meyer-Olkin, bem como o teste de esfericidade de Bartllet indicaram que os dados são apropriados para análise fatorial
$\left(\mathrm{KMO}=, 770, \quad \mathrm{X}^{2}=825,5\right.$ para os homens $\mathrm{e}$ $\mathrm{KMO}=, 859, X^{2}=963,2$ para as mulheres, ambos com $\mathrm{p}<, 001)$. Assim, a análise fatorial exploratória 
para os homens, demonstrou que apenas 13 desses itens tinham alta carga (pelo critério $r$ $>, 500$ ) em um dos três fatores, sendo que o primeiro fator (Imagem Corporal) explica 13\% da variância total, o segundo fator (Catexia Corporal) explica $13,6 \%$ e 0 terceiro (satisfação com o peso) responde por $31,1 \%$. Em relação às mulheres os 14 itens tiveram alta carga (pelo critério $r>.500$ ) nos três fatores, sendo que o primeiro fator (Imagem Corporal) explica 9,9\% da variância total, o segundo (Catexia Corporal) explica $13,2 \%$ e o terceiro (satisfação com o peso) responde a $40,5 \%$.

Além disso, obteve-se um alfa de Cronbach total de, 757 , em que os homens apresentaram $\alpha=, 780$ e as mulheres $\alpha=, 758$.

\section{Discussão}

\section{Satisfação Corporal de Acadêmicos de Educação Física}

Observou-se que, em geral, os participantes estavam de médio a muito satisfeitos com seus corpos. Estes dados podem ser considerados positivos, uma vez que a satisfação corporal pode refletir em uma boa aparência e autoconfiança, minimizando a transferência das insatisfações e medos para o conjunto social e/ou profissional. Assim, de acordo com Tiggemann (2004), a conquista da satisfação corporal pode proporcionar confortáveis condições sócio-psicoculturais. Tal achado foi, igualmente, evidenciado por Bossi et al (2006) ao avaliarem a autopercepção corporal de estudantes brasileiras de nutrição. Os resultados encontrados confirmam que as mesmas desejam adequar seus corpos ao padrão social vigente, mesmo ao apresentarem peso apropriado.

Em um estudo realizado no Brasil por Kakeshita e Almeida (2006), avaliando o índice de massa corporal e a auto-imagem de universitários, concluíram que homens e mulheres subestimam-se ou superestimam-se quanto à imagem corporal. Gaiarsa (1984) afirma que o indivíduo não conversa com seu próprio corpo, sugerindo dissociações entre o percebido e o real, quanto à imagem corporal e o seu próprio Eu, isto pode explicar as possíveis distorções as quais nos referimos.

Em contrapartida, um estudo com universitários chineses demonstrou que, em geral, homens e mulheres estavam insatisfeitos com o peso e com o corpo, sendo que houve correlação significativa entre sintomas depressivos e insatisfação corporal somente nas mulheres (DAVIS; KATZMAN, 1997).
Em relação ao sexo, algumas peculiaridades foram encontradas para a satisfação corporal: os homens deste estudo "gostavam mais da cor da pele", "achavam seus corpos mais proporcionais" e "estavam mais satisfeitos com seus corpos" comparativamente às mulheres. $\mathrm{Da}$ mesma forma, estudos sobre a satisfação corporal têm demonstrado que as mulheres universitárias são menos satisfeitas com seus corpos que os homens (DAVIS; KATZMAN, 1997). Nesse sentido, um estudo sugere que as meninas a partir dos 6 anos de idade (pré-adolescência) já começam a se preocupar com a imagem corporal e a sentirem insatisfação com o próprio corpo (DOHNT; TIGGEMANN, 2006). Nesse período, a insatisfação com o corpo tende a aumentar significativamente nas meninas e a diminuir nos meninos (DOHNT; TIGGEMANN, 2006, BEARMAN et al., 2006).

As mulheres do estudo, em média, "olhavam seus corpos no espelho", "se auto-massageavam" e "tocavam seus corpos" com mais freqüência. Neste processo, elas "percebiam que os outros as achavam sexys" e "queriam ser mais magras" que os homens do estudo. De acordo com Tiggemann (2004), muitas mulheres aceitam e buscam o padrão de beleza da mulher magra e essa busca pode gerar sofrimentos e conflitos em muitas delas. Se comparadas aos homens, as mulheres se preocupam mais com a aparência física, são mais predispostas a se perceberem mais pesadas, são mais insatisfeitas com seus corpos e desejam perder peso constantemente (KASHUBECK-WEST et al., 2005). Possivelmente, tais mulheres estão mais preocupadas com a percepção do outro e com o feedback social que os homens. De acordo com Lee et al. (2009) o feedback social exerce forte influência nas crenças, atitudes e comportamentos relacionados ao corpo.

A satisfação com o peso, por parte dos homens, esteve positivamente correlacionada $(r=, 328, p<, 001)$ com "o quanto se considerava fisicamente ativo", tal comportamento enfatiza a importância do exercício enquanto mantenedor da satisfação com o peso. MacCabe e Ricciardelli (2004) postulam que a insatisfação corporal em homens tem sido associada com problemas alimentares, uso de esteróides e também com dependência à prática de exercício físico. Outro estudo, com homens adolescentes praticantes de futebol, demonstrou a importância de apresentar uma boa forma física (magra) para a imagem corporal e auto-estima destes (MACKINNON et al., 2003). Além disso, foi verificado que os homens mesmo estando mais satisfeitos com 
seus corpos são mais predispostos a procurar exercício físico (FROST; MACKELVIE, 2005).

Obtiveram-se correlações positivas entre a idade e os três índices de satisfação corporal tanto para homens quanto para mulheres. Corroborando a isso, Esnaola et al (2009) estudando a insatisfação corporal de homens e mulheres em diferentes faixas etárias, verificou que entre as participantes do sexo feminino as adolescentes apresentam maior insatisfação corporal e são mais afetadas pela publicidade e modelos sociais quando comparadas às mulheres mais velhas. Além disso, Oberg e Tornstam (1999), também verificaram que com o aumento da idade não ocorria queda da satisfação corporal, como o previsto por eles, confirmando a menor preocupação das mulheres mais velhas com a aparência.

As três dimensões do construto mostraram-se mais correlacionadas entre si para o grupo de mulheres, o que pode indicar que esse modelo seja mais adequado à percepção feminina do conceito. Frost e Mackelvie (2005) também encontraram correlação positiva entre catexia corporal e imagem corporal, mas para ambos os sexos $(, 339, p<, 001)$.

\section{Validação de Construto e Confiabilidade Interna do Questionário}

A partir da análise das diferenças entre homens e mulheres e confrontando-as com a literatura, pode-se observar que o instrumento foi sensível para captar algumas diferenças entre os sexos já mencionadas em outros estudos.

Havendo, pois, (1) base teórica suficiente para o construto, (2) análise de fatores ter detectado os fatores principais e (3) tendo o instrumento aqui utilizado detectado as diferenças entre homens e mulheres, compreende-se que este instrumento é válido e confiável para avaliar o construto Satisfação Corporal.

Entende-se que o presente estudo tenha apresentado limitações referentes ao tempo reduzido de coleta, à aplicação ter sido feita apenas em uma amostra de estudantes do curso de Educação Física e o processo de validação do questionário estar sendo concluído.

Apêndice: Escala de Satisfação Corporal

\begin{tabular}{|c|c|c|c|c|c|c|c|}
\hline \multirow[b]{2}{*}{ O quanto olha seu corpo inteiro no espelho } & \multicolumn{3}{|c|}{ Pouco } & \multirow{2}{*}{$\begin{array}{c}\text { Médio } \\
3\end{array}$} & \multicolumn{3}{|c|}{ Muito } \\
\hline & 0 & 1 & 2 & & 4 & 5 & 6 \\
\hline O quanto se auto-massageia & 0 & 1 & 2 & 3 & 4 & 5 & 6 \\
\hline O quanto toca seu corpo de forma geral & 0 & 1 & 2 & 3 & 4 & 5 & 6 \\
\hline O quanto gosta do seu cabelo & 0 & 1 & 2 & 3 & 4 & 5 & 6 \\
\hline O quanto gosta da cor dos seus olhos & 0 & 1 & 2 & 3 & 4 & 5 & 6 \\
\hline O quanto gosta da cor da sua pele & 0 & 1 & 2 & 3 & 4 & 5 & 6 \\
\hline O quanto gosta do seu rosto & 0 & 1 & 2 & 3 & 4 & 5 & 6 \\
\hline O quanto percebe que causa uma boa impressão & 0 & 1 & 2 & 3 & 4 & 5 & 6 \\
\hline O quanto acha seu corpo bonito & 0 & 1 & 2 & 3 & 4 & 5 & 6 \\
\hline O quanto acha seu corpo proporcional & 0 & 1 & 2 & 3 & 4 & 5 & 6 \\
\hline O quanto acha seu corpo sensual & 0 & 1 & 2 & 3 & 4 & 5 & 6 \\
\hline O quanto está satisfeito com seu corpo & 0 & 1 & 2 & 3 & 4 & 5 & 6 \\
\hline O quanto percebe que os outros o acham sexy & 0 & 1 & 2 & 3 & 4 & 5 & 6 \\
\hline O quanto gostaria de ser mais magro & 0 & 1 & 2 & 3 & 4 & 5 & 6 \\
\hline
\end{tabular}

\section{Conclusão}

Dado o exposto, conclui-se que o construto Satisfação Corporal pode ser avaliado a partir da Escala de Satisfação Corporal apresentada no presente trabalho (APÊNDICE), considerando-se as três dimensões do modelo de Frost e McKelvie (2005): Imagem Corporal, Catexia Corporal e Satisfação com o Peso. Deve-se enfatizar que tal instrumento deriva destes pressupostos teóricos, sendo útil porque é objetivo e de fácil aplicação.

Seria prudente, em uma etapa futura, ampliar e testar o uso desse instrumento, mediante a aplicação em populações de diferentes idades, o que permitirá comparações com outras categorias profissionais que lidam, igualmente, com a interface corpo-satisfação. 


\section{Referências}

ATA, R.; LUDDEN, A. B.; LALLY, M. M. The effects of gender and family, friend, and media influences on eating behaviors and body image during adolescence. Journal of Youth and Adolescence, Bloomington, v. 36, p. 1024-1037, 2007.

BEARMAN, S. K.; MARTINEZ, E.; STICE, E.; PRESNELL, K. The skinny on body dissatisfaction: a longitudinal study of adolescent girls and boys. Journal of Youth and Adolescence, Bloomington, v. 35, n. 02, p. 217229, abr. 2006.

BOSSI, M. L. M.; LUIZ, R. R.; MORGADO, C. M. C.; COSTA, M. L. S. C.; CARVALHO, R. H. Autopercepção da imagem coporal entre estudantes de nutrição: um estudo no município do Rio de Janeiro. Jornal Brasileiro de Psiquiatria, São Paulo, v. 5, n. 2, p. 108-113, 2006.

CARDOSO, F. L.; SAVALL, A. C.; SABBAG, S.; MENDES, A. K.; BELTRAME, T. S. Implicações do conhecimento corporal no comportamento sexual. Revista Brasileira de Educação Física e Esporte, São Paulo, v. 23, n. 4, p. 345-354, out. 2009.

CARDOSO, F. L.; SILVEIRA, R. A.; ZEQUINÃO, M. A.; MARTINS, C.; SOUZA, C. A. Autopercepção corporal e preferências motoras de praticantes de dança. Movimento, Porto Alegre, v. 16, n. 1, p. 97-112, jan. 2010.

DAVIS, C.; KATZMAN, M. Charting new territory: body esteem, weight satisfaction, depression, and self-esteem among chinese males and females in Hong Kong. Sex Roles, Pittsburgh, v. 36, n. 7, p. 449-459, apr. 1997.

DOHNT, H. K.; TIGGEMANN, M. Body image concerns in young girls: the role of peers and media prior to adolescence. Journal of Youth and Adolescence, Bloomington, v. 35, n. 2, p. 141-151, apr. 2006.

ESNAOLA, I.; RODRÍGUEZ, A.; GOÑI, A. Body dissatisfaction and perceived sociocultural pressures: gender and age differences. Salud Mental, México, v. 33, n. 1, p. 21-29, jan./fev. 2010

FROST, J.; MCKELVIE, S. The relationship of self-esteem and body satisfaction to exercise activity for male and female elementary school, high school, and university students. Sex Roles, Pittsburgh, v. 51, n. 2, p. 45-54, jul. 2004.

GAIARSA, J. A. O espelho mágico: um fenômeno chamado corpo e alma. 13. ed. São Paulo: Summus, 1984

HARGREAVES, D. A.; TIGGEMANN, M. Idealized media images and adolescent body image:

"comparing" boys and girls. Body Image, Norfolk, v. 1, p. 351-361, dez. 2004.

HUANG, J. S.; NORMAN, G. J.; ZABINSKI, M. F.; CALFAS, K.; PATRICK, K. Body image and selfesteem among adolescents undergoing an intervention targeting dietary and physical activity behaviors. Journal of Youth and Adolescence, Bloomington, v. 40, n. 3, p. 245-251, mar. 2007.

JONES, D. C. Body image among adolescent girls and boys: a longitudinal study. Developmental Psychology, Ann Arbor, v. 40, p. 823-835, set. 2004.

JUNG, J.; LENNON, S. J.; RUDD, N. A. Selfschema or self-discrepancy?: which best explains body image? Clothing and Textiles Research Journal, Thousand Oaks, v. 19, n. 4, p. 171-184, jun. 2001.

KAKESHITA, I. S, ALMEIDA, S. S. Relação entre índice de massa corporal e a percepção da autoimagem em universitários. Revista de Saúde Pública, São Paulo, v. 40, n. 3, p. 497-504, 2006.

KASHUBECK-WEST, S.; MINTS, L. B.; WEILGOLD, I. Separating the effects of gender and weight-loss desire on body satisfaction and disordered eating behavior. Sex Roles, Pittsburgh, v. 53, n. 7, p. 505-518, out. 2005.

KOSTANSKI, M.; GULLONE, E. Adolescent body image dissatisfaction: relationships with selfesteem, anxiety, and depression controlling for body mass. The Journal of Child Psychology and Psychiatry, Southampton, v. 39, p. 225-262, dez. 1998.

LEE, H.; DAMHORST, M. L.; OGLE, J. P. Body satisfaction and attitude theory: linkages with normative compliance and behaviors undertaken to change the body. Family and Consumer Sciences Research Journal, Alexandria, v. 37, n. 4, p. 466-488, mar. 2009.

LOWERY, S. E.; KURPIUS, S. E. R.; BEFORT, C.; BLANKS, E. H.; SOLLENBERGER, S.; NICPON, M. F.; HUSER, L. Body image, self- 
esteem, and health-related behaviors among male and female first-year college students. Journal of College Student Development, Nashville, v. 46, n. 6, p. 612-623, nov./dez. 2005.

MCCABE, M. P.; RICCIARDELLI, L. A. Body image dissatisfaction among males across the lifespan: a review of past literature. Journal of Psychosomatic Research, Kennesaw, v. 56, p. 675-685, 2004.

MACKINNON, D. P.; GOLDBERG, L.; CHEONG, J.; ELLIOT, D.; CLARKE, G.; MOE, E. Male body esteem and physical measurements: do leaner, or stronger, high school football players have a more positive body image? Journal of Sport \&

Exercise Psychology, Tallahassee, v. 25, p. 307322, jul. 2003.

NEUMAN, D. L.; SONTAG, L. M.; SALVATO, R. Psychosocial aspects of body mass and body image among rural american indian adolescents. Journal of Youth and Adolescence, Bloomington, v. 35, n. 2, p. 281-291, abr. 2006.

OBERG, P.; TORNSTAM, L. Body images among men and women of different ages. Ageing and Society, Cambridge, v. 19, p. 629-644, 1999.

PETROSKI, E. L.; PELEGRINI, A.; GLANER, M. $F$. Insatisfação corporal em adolescentes rurais e urbanos. Motricidade, Vila Real, v. 5, n. 4, p. 1325, set. 2009.

PHILIPS, J. M.; DRUMMOND, M. J. N. An investigation into the body image perception, body satisfaction and exercise expectations of male fitness leaders: implications for professional practice. Leisure Studies, Abingdon, v. 20, p. 95105, maio 2001.

PUHL, R.; BROWNELL, K. D. Obesity, bias, and discrimination. Obesity Research, Los Angeles, v. 8 , p. $788-805$, set. 2001.

RICCIARDELLI, L. A.; MCCABE, M. P. Sociocultural and individual influences on muscle gain and weight loss strategies among adolescent boys and girls. Psychology in the Schools, Hoboken, v. 40, p. 209-224, jan. 2003.

SACOMORI, C.; CARDOSO, F. L.; VANDERLINDE, C. Pelvic floor muscle strength and body self-perception among Brazilian pregnant women. Physiotherapy, Hawthorn, v. 96, abr. 2010. In press.
STICE, E.; ANDWHITTENTON, K. Risk factors for body dissatisfaction in adolescent girls: a longitudinal investigation. Developmental Psychology, Ann Arbor, v. 38, p. 669-678, nov. 2002.

TAVARES, M. C. G. C. F. Imagem corporal: conceito e desenvolvimento. Barueri: Manole, 2003.

TIGGEMAN, M. Body image across the adult life span: stability and change. Body image, Norfolk, v. 1, p. 29-41, nov. 2004.

TIGGEMAN, M.; WILLIAMSON, S. The effect of exercise on body satisfaction and self-esteem as a function of age and gender. Sex Roles, Pittsburgh, v. 43, n. 2, p. 119-127, jul. 2000.

VANDERWAL, J. S.; THELEN, M.H. Eating and body image concerns among obese and averageweight children. Addictive Behaviors, Arlington, v. 25 , p. $775-778$, set./out. 2000.

Endereço:

Fernando Luiz Cardoso

CEFID - UDESC.

Rua Paschoal Simone, 358 Coqueiros

Florianópolis SC Brasil

88080-350

Telefone: (48) 3321.8600

e-mail: fernandocardoso.ph.d.lagesc@gmail.com Homepage: $\underline{\text { http://www.cefid.udesc.br/?id=633 }}$

Recebido em: 06 de julho de 2010.

Aceito em: 27 de dezembro de 2011.

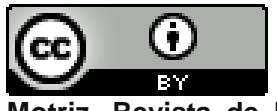

Motriz. Revista de Educação Física. UNESP, Rio Claro, SP, Brasil - elSSN: 1980-6574 - está licenciada sob Creative Commons - Atribuição 3.0 